\title{
Kernos
}

Revue internationale et pluridisciplinaire de religion grecque antique

$24 \mid 2011$

Varia

\section{Claude Calame, Prométhée généticien. Profits techniques et usages de métaphores}

\section{Aurian Delli Pizzi}

\section{OpenEdition \\ Journals}

\section{Édition électronique}

URL : http://journals.openedition.org/kernos/1973

DOI : 10.4000/kernos. 1973

ISSN : 2034-7871

\section{Éditeur}

Centre international d'étude de la religion grecque antique

\section{Édition imprimée}

Date de publication : 1 janvier 2011

Pagination : $339-341$

ISSN : 0776-3824

\section{Référence électronique}

Aurian Delli Pizzi, «Claude calame, Prométhée généticien. Profits techniques et usages de métaphores », Kernos [En ligne], 24 | 2011, mis en ligne le 17 octobre 2011, consulté le 21 septembre 2020. URL : http://journals.openedition.org/kernos/1973 ; DOI : https://doi.org/10.4000/kernos.1973 
CALAme Claude, Prométhée généticien. Profits techniques et usages de métaphores, Paris, Les Belles Lettres; Encre Marine, 2010. 1 vol. 11,5 × 17 cm, 204 p. (Collection "À présent »). ISBN : 978-2-35088-022-8.

S'il est des études susceptibles d'embarrasser les détracteurs de nos disciplines, selon lesquels nos champs d'investigation n'ont aucune «utilité » pour comprendre et analyser le monde contemporain, celle-ci en fait partie. Dans cet ouvrage original et d'une grande acuité, qualifié d' «essai» (p. 13), C.C. se fonde sur une interprétation sémiotique des textes grecs anciens pour étudier différentes questions épistémologiques relatives à la génétique moderne. L'A. entend appréhender le mythe de Prométhée, point d'ancrage de son analyse, non seulement à travers sa version "canonique» tel qu'il se décline dans la Théogonie d'Hésiode, mais surtout dans la version plus « sophistique » présentée dans le Prométhée enchainé d'Eschyle. Cette perspective vise à proposer une lecture de la tragédie eschyléenne non pas dans une vision progressiste, qui insiste sur le fait que Prométhée a conduit les hommes de l'ignorance à la connaissance, mais dans une dimension sémiotique et interprétative, pour mieux saisir les enjeux qui sous-tendent la démarche prométhéenne de transmission des arts et techniques aux humains. Cette relecture se double d'une comparaison critique avec le champ de la génétique « dans les termes de la neurobiologie très naïve et fruste d'un praticien des sciences humaines » (p. 85). C.C. part en effet de l'hypothèse que les techniques offertes par Prométhée à l'homme démuni sont comparables à l'espoir qui découle du génie génétique en termes de progrès. La pierre angulaire de cette comparaison est la notion d' " anthropopoiésis », à savoir la fabrication de l'homme par lui-même, en interaction avec son environnement et ses pairs. En outre, cette comparaison doit permettre une remise en question des métaphores généralement utilisées pour qualifier et appréhender la génétique.

S’il est vrai que la réflexion de C.C. s'articule en trois étapes, à savoir les arts prométhéens de déchiffrement des signes, la construction culturelle de l'homme par lui-même et la sémiotique du génie génétique contemporain - l'A. qualifie lui-même son essai de «triptyque» (p. 21) l'ouvrage est en réalité composé de cinq chapitres: "Les tékhnai de Prométhée et la condition humaine », «Inachèvement de l'homme et procédures d'anthropopoiésis », "Anthropopoiésis par le génie génétique: déterminismes en question», "Aléatoire herméneutique et utilité sociale » et «Renaissance des sciences humaines ». Je reprends ici les principales lignes de force de l'exposé.

Les techniques offertes par Prométhée constituent avant tout un savoir-faire d'ordre interprétatif, puisque les mekhanémata, sophismata et ophelémata (termes utilisés par Eschyle et repris par l'A.) qu'a offerts Prométhée à travers son acte d'bybris sont destinés à permettre à tous les hommes sans exception de jouir d'une plus grande autonomie matérielle en exploitant les capacités sensorielles qu'ils n'étaient guère en mesure d'utiliser jusqu'alors, afin de communiquer avec les dieux et avec leurs pairs. Par ailleurs, une comparaison entre le célèbre passage du Protagoras de Platon sur la création de l'espèce humaine, où les arts pratiques prométhéens s'inscrivent dans l'ordre de la justice politique accordée par Zeus, et l'Antigone de Sophocle, où sont abordées les limites assignées aux hommes par les dieux, permet de soulever la question de l'inné et de l'acquis dans la nature humaine, laquelle constitue un des fils conducteurs de l'ensemble de l'ouvrage. Dans tous les cas, le destin auquel sont soumis tous les acteurs, tant divins qu'humains, joue un rôle primordial et empêche de voir Prométhée comme un philosophe libre de ses mouvements, ce que suggère, à tort, la lecture teintée d'idéologie nazie d'Heidegger.

Tant la pensée grecque classique que le romantisme allemand (C.C. prend l'exemple de Herder) insistent sur le fait que les qualités innées de l'homme peuvent être développées par son intelligence, à une différence près : il n'y a guère, selon le romantisme allemand, de Zeus tout-puissant et de destin auxquels l'homme est assujetti, mais plutôt une nature unique créée 
par un dieu unique. L'homme, par nature inachevé, peut toutefois dépasser et compenser son incomplétude. C.C. s'intéresse aux apports de la neurologie sur la question, qui ont montré la plasticité de nos facultés cérébrales face à ce que l'on peut appeler la «culture », en opposition à la nature. En d'autres termes, le cerveau humain est constamment façonné par l'environnement auquel il est soumis. À cette plasticité neurologique s'ajoute une plasticité corporelle, sur laquelle l'homme peut jouer pour se définir, comme l'attestent les actes pratiqués dans différentes cultures (régime alimentaire, usages de la voix, ornements esthétiques, etc.). En définitive, cette "auto-fabrication ", qu'elle s'opère par le biais des techniques interprétatives offertes par Prométhée ou de tout autre méthode, ne doit pas être conçue comme un moyen de combler une lacune, un inachèvement, mais bien comme une construction positive de l'être humain. C.C. pose alors la question de savoir si les importants moyens financiers dévolus à l'ingénierie génétique poursuivent un projet civilisateur à l'instar des tékhnai de Prométhée. S'il est vrai que, indéniablement, la génétique permet des progrès dans le domaine thérapeutique, la frontière entre traitement positif, où l'on «soigne » l'humain pour le faire correspondre à un idéal, et négatif, où l'on cherche à éviter les handicaps, est relativement poreuse. De surcroît ces traitements sont l'apanage des plus nantis.

La critique de C.C. porte également sur les principes épistémologiques impliqués par certaines métaphores couramment utilisées, principalement celle de «déterminisme biologique » et de "programme génétique ». La manipulation génétique ne suit pas un simple principe de cause à effet, tel qu'il a été défendu par Crick et Watson, mais s'opère en interaction avec toute une série de facteurs, impliquant une certaine marge d'incertitude et d'aléatoire. Tout aussi trompeur est le concept de "patrimoine génétique ». Le modèle du «code » à déchiffrer et à reproduire est également inexact, à tel point que cloner un être vivant à l'identique relève du pur fantasme de l'être humain aspirant à l'immortalité et s'avère impossible en pratique. Dans la réalité, il s'agit davantage de clonage thérapeutique, où l'on produit des cellules souches, que de clonage reproductif.

C.C. insiste également sur la grande part d'aléatoire dans les processus génétiques. Dès lors surgit cette question : si l'homme peut se substituer à Zeus ou à Prométhée, peut-il, en définitive, se substituer au hasard - donnée rarement prise en compte par les biologistes ? L'bybris de l'homme est en réalité double, puisque, en plus de refuser de reconnaittre son incapacité face au hasard, il applique des technologies implicitement fondées sur des erreurs telles qu'un clivage artificiel entre inné et acquis, nature et culture, etc.

Derrière cette comparaison critique, on voit apparaitre, régulièrement, les prises de position de C.C. contre le néolibéralisme. Tout en égratignant au passage plusieurs acteurs politiques, économiques ou scientifiques, C.C. évite toutefois habilement de tomber dans le piège de la critique purement négative du clonage. Il dénonce d'ailleurs la critique trop souvent faite à la génétique, et largement liée à notre héritage judéo-chrétien, selon laquelle il faut condamner la volonté de l'homme de jouer à Dieu. La principale critique de C.C. à l'encontre des manipulations génétiques porte sur la logique du pur profit, héritée de l'économie néolibérale et capitalisée, qui guide les recherches génétiques sans porter d'intérêt aux avantages sociaux. En définitive, l'exemple de Prométhée nous montre qu'il faudrait considérer l'ingénierie génétique comme un art pratique et interprétatif et non comme technologie source de profits financiers. Il ne faut néanmoins pas contraster exagérément l'image d'un Prométhée offrant des ressources pour le bien de l'humanité entière et la technologie génétique contemporaine négligeant l'homme : le respect de la dignité humaine tel qu'elle se décline dans la Déclaration Universelle des Droits de l'Homme, dans laquelle les droits de l'individu l'emportent sur le seul intérêt de la science ou de la société, peut certes s'opposer aux critères de l'économie néolibérale, mais elle ne correspond pas pour autant à la conception de l'humain telle qu'elle ressort du texte d'Eschyle, dans lequel c'est la société, non l'individu, qui prime. 
Il ne m'appartient pas de juger les connaissances de C.C. en termes de neurologie et de génétique, mais force est de constater que l'aisance et la précision dont il fait preuve en ces domaines sont remarquables. De surcroit, dans la mesure où il est question avant tout de principes épistémologiques, le propos ne porte généralement pas sur des questions techniques qui seraient inaccessibles aux non initiés. D'autre part, le but de cet ouvrage, comme tout essai, est de stimuler le débat, sans viser l'exhaustivité, et c'est dans cette optique qu'il doit être lu. Le lecteur ne manquera donc pas de soulever des questions par lui-même. Par exemple, la critique globale de la subordination de la génétique au profit financier permet difficilement de se faire une idée précise des conditions de travail des scientifiques dans le domaine de la recherche universitaire. Il reste donc à voir la portée que cette réflexion aura, d'un point de vue pragmatique, sur les chercheurs en génétique eux-mêmes. De plus, le parallèle entre les apports prométhéens et la génétique est tout à fait fondé, à cette divergence près : le récit concernant Prométhée s'opérant « après coup », les techniques qu’il a transmises sont jugées indispensables à l'homme - qui ne conçoit pas, par exemple, de vivre sans feu. Le cas de la génétique pose un problème différent, puisque nous ne savons pas encore jusqu'où cette technique va nous mener. Mais sans doute est-ce justement cette différence qui est intéressante. C.C. ouvre la voie à un débat que l'avenir et les incessants progrès technologiques rendront peut-être encore plus inévitable, tout en utilisant des outils inattendus mais néanmoins pertinents : les textes grecs anciens.

Aurian Delli Pizzi

(F.R.S.-FNRS - Université de Liège)

Giovanni TOSETTI, Unioni divino-umane. Un percorso storico-religioso nel mito greco arcaico, Cosenza, Edizioni Lionello Giordano, 2008. 1 vol. 14,5 × 20,5 cm, vii+515 p. (HIER A. Collana di studi storico-religiosi, 10). ISBN : 978-88-86919-26-3.

Cet ouvrage est issu d'une thèse de Doctorat en Histoire religieuse que l'A. a soutenue à l'Université de Messina (Italie) en 2003 et représente une importante étape d'un parcours de recherche que l'A. a poursuivi aussi par la suite ${ }^{1}$. Comme Concetta Scibona Giuffré le signale dans la préface, l'A. est l'« estrema propaggine della scuola di U. Bianchi», dont l'héritage est bien présent dans la perspective scientifique adoptée dans ce livre. L'originalité de la démarche consiste, quant à elle, dans la tentative de mettre en regard cet héritage avec les interprétations $\mathrm{du}$ « mythe » et des pratiques discursives des Grecs qui ont été offertes ces dernières décennies par, et dans le sillage de, Marcel Detienne et Claude Calame. Le cadre méthodologique est précisé dans l'introduction : par l'analyse des récits d'époque archaïque qui thématisent la fin de l'âge héroïque et, avec elle, de la race des demi-dieux, l'A. se propose de vérifier l'existence, en Grèce ancienne, d'un patrimoine de récits qui pourraient à bon droit être définis comme « mythes ». Afin de démontrer la pertinence de la catégorie de « mythe », de plus en plus remise en question aussi par les hellénistes, l'A. se propose d'identifier dans les sources littéraires analysées une série de traditions irréductibles aux circonstances énonciatives particulières. Le noyau thématique stable de ces traditions véhiculerait la vision que les Grecs d'époque archaïque avaient développée d'une époque révolue, l’âge héroïque, caractérisée par une temporalité spécifique et par l'intimité des relations entre les dieux et les hommes. Le «mythe » dont l'A. suit les traces est plus particulièrement celui qui concerne à la fois la naissance des demi-dieux, descendants directs (mais aussi indirects) des unions entre immortels et mortels, et leur disparition, programmée par les dieux, à la suite des guerres de Thèbes et de Troie. Tout au long de la démonstration, le mot «mythe » est systématiquement remplacé par «le temps raconté », dont

1 Cf. G. TOSETTI, «La dernière génération héroïque : un parcours historico-religieux et sémio-narratif, d'Hésiode au ps-Apollodore », Kernos 19 (2006), p. 113-130. 